\title{
MUSEOS DE ARTE Y EDUCACIÓN, ALIANZAS ENTRE MUSEOS Y DOCENTES DE EDUCACIÓN ARTÍSTICA
}

\author{
Natalia MIRALLES JARA ${ }^{1}$
}

RESUMEN: El siguiente trabajo se centra en el ámbito de la educación de las artes visuales en Santiago de Chile y la forma en que los docentes de esta asignatura se relacionan con la labor educativa realizada por los museos de arte.

Actualmente la escuela ha ido perdiendo protagonismo en su tarea de educar, ya no es concebida como el gran núcleo del saber debido a la masificación del acceso a la web, los medios de comunicación y al surgimiento de iniciativas en el ámbito no formal e informal que son decidoras en el proceso de enseñanza-aprendizaje de los estudiantes. Dentro de estas instituciones podemos encontrar al Museo de Arte, el cual ha comenzado a desarrollar una labor educativa muy potente dentro de las últimas décadas.

La investigación es producto del Seminario de Grado Museos de Arte y Educación, alianzas entre museos y docentes de educación artística ${ }^{2}$ y se centra en estudiar la relación que existe entre el museo de arte y educación artística en el ámbito escolar. Para establecer cuáles son estos nexos, se utiliza una metodología de enfoque cualitativo, a través de la recopilación de información mediante entrevistas semi- estructuradas a cuarenta docentes de artes visuales, y a ocho museos de Santiago de Chile, más tres instituciones culturales que han desarrollado un gran aporte a la educación artística no formal. Los resultados obtenidos se analizan de forma interpretativa-descriptiva, con el fin de realizar conclusiones significativas sobre el nexo real entre los docentes de artes y las instituciones museísticas y culturales.

PALABRAS CLAVES: Educación artística. Educación no formal. Arte. Museos.

\section{Introducción}

El interés por investigar en torno a la potencial alianza entre los museos de arte y los docentes del área artística surge desde la observación de lo que acontece dentro de nuestras aulas en la clase de artes visuales.

En Chile es evidente el capital cultural desigual de los estudiantes, en donde alumnos de clases sociales más altas son los que poseen más cercanía con las expresiones artísticas, teniendo un mayor conocimiento de conceptos relacionados al arte, visitando constantemente instituciones culturales y museos y en definitiva desarrollando de mejor forma competencias culturales y artísticas.

\footnotetext{
${ }^{1}$ Licenciada en Artes Visuales. Universidad de Chile; Profesora de Artes Visuales. Pontificia Universidad Católica de Chile; Doctora en Bellas Artes. Universidad Complutense de Madrid. Académica Departamento de Artes. Universidad Católica Raul Silva Henríquez. San Isidro - Santiago - Chile. 6500620 - nmiralles@ucsh.cl.

${ }^{2}$ El seminario se realiza como requisito para la obtención del título en la carrera de Pedagogía en Educación Artística en la Universidad Católica Silva Henríquez. Este fue dirigido por la autora y los alumnos participantes fueron: Ángela Córdova, Gerar Mancilla, Scarlette Sánchez, Luz María Sanhueza, y Felipe Trujillo.
} 
Muy por el contrario a la realidad de estudiantes pertenecientes a un grupo de la población de bajos recursos, donde la analfabetización visual, el desconocimiento artístico, poco bagaje cultural y el desinterés por el ámbito artístico-cultural son hechos muy comunes y hacen que la brecha en este ámbito sea cada día mayor en nuestra sociedad.

Esto se relaciona con lo que señala Bourdieau (2002), en donde el poder del capital cultural y los centros o sectores poseedores del mismo, han procurado trasmitir este saber hacia sus descendientes, manteniendo este conocimiento dentro de un mismo círculo de élite.

Con las diversas reformas que se han realizado al sistema educativo, reconociendo a la escuela como un poseedor y trasmisor del capital cultural, se puede considerar la alfabetización visual como un medio de democratización cultural que puede llevar a cabo el subsector de Artes Visuales.

Revisando los planes y programas del área podemos notar como éstos sugieren visitar instituciones culturales como el museo, con el fin de complementar los procesos de enseñanza-aprendizaje. Esta directriz reconoce el poder de los escenarios no formales de educación y tiene como objetivo principal potenciar el contacto directo con la obra, para instar que el público escolar tenga experiencias que les permitan entre otras cosas desarrollar fenómenos como: descubrimiento, apreciación estética, pensamiento crítico y capacidad de análisis en torno a la visualidad.

De la misma manera con los años, los museos de arte también se han abierto a la función educativa, reconociendo en esta un pilar de su labor como institución dedicada a la preservación y difusión del patrimonio artístico. Se han creado departamentos educativos que implementan iniciativas específicas para los visitantes escolares; incluyendo material didáctico, visitas guiadas, diferenciación de actividades por nivel de enseñanza y capacitaciones docentes entre otras. Utilizando como estrategia principal la creación de conexiones entre los programas de estudio y las colecciones del museo para generar iniciativas que los docentes puedan considerar como apoyos en su tarea de enseñar arte.

De esta forma el museo siendo un espacio educativo no formal puede conectarse de forma sinérgica con el aprendizaje que se lleva a cabo en la escuela generando expectación, ansiedad y motivación del alumnado por el hecho de contemplar directamente una obra, vivencia que en muchos de los casos sólo es posible en este contexto ya que no existe el hábito del ocio cultural en gran parte de las familias chilenas. 
Sin embargo a pesar de este panorama auspicioso la realidad nos muestra un escenario un poco distinto, y hoy observamos una realidad que difiere de la proyección establecida, en donde todavía no se logra afianzar la relación entre ambas instituciones.

Para entender este proceso y el actual estado en que nos encontramos analizaremos el desarrollo de la función educativa en los museos, tomando como punto de referencia la actualidad chilena al respecto y la forma en que se ha generado el intercambio entre Museo y Escuela.

\section{Descripción del trabajo}

Para abordar el tema es relevante comprender la evolución que han tenido los museos, específicamente desde mediados del siglo XX donde se gestan los cambios más significativos que promueven la importancia de una función educativa al interior de estas instituciones.

Es en 1946 con la creación de ICOM (International Council of Museums) la función educativa se reconoce como un eje relevante, lo cual queda de manifiesto en la propia definición de la institución museal: “[...] Un museo es una institución permanente, sin fines de lucro, al servicio de la sociedad y abierta al público, que adquiere, conserva, estudia, expone y difunde el patrimonio material e inmaterial de la humanidad con fines de estudio, educación y recreo". (ICOM, 2010).

Posteriormente esto se afianza gracias al desarrollo de la Nueva Museología que propone cambiar la noción tradicional del museo como ente destinado a conservar patrimonio y convertirlo en una institución abierta y dialogante que propicie la participación de la comunidad. Afianzando la noción de que los museos dejan de ser solo “guardianes" sino también espacios de participación activa y crítica.

En Chile todavía existe un escenario incipiente en relación al contexto internacional, el cual lleva un mayor período de reflexión y análisis en torno a la pedagogía museística. A pesar del retraso, nuestro país no se encuentra ajeno al desarrollo museístico y es por esto que a un lustro del s. XXI es interesante investigar cómo han evolucionado los museos chilenos en este ámbito y también la forma en que los docentes de artes visuales perciben a los museos y los potenciales usos pedagógicos que pueden hacer de sus colecciones. 
Para ello se centra la investigación en ocho Museos de Arte de Santiago de Chile y cuarenta docentes del área artística que realicen o hayan realizado trabajo escolar dentro de la misma localidad, analizando sus discursos y la realidad práctica que presentan ambos agentes a nivel educativo.

\section{Metodología}

La investigación se enmarca dentro del enfoque cualitativo lo cual permite que se aborde el fenómeno de forma holística, realizando una recolección de datos no es estandarizada que permite obtener perspectivas y puntos de vistas amplios en torno al tema en cuestión.

Al introducirnos en el contexto nacional sobre la relación entre museos de arte y docentes de arte, es necesario focalizar la atención en ambas áreas para recolectar datos, analizarlos y obtener conclusiones, estableciendo ambos contextos como objetos de estudio.

El escenario de estudio es Santiago de Chile, quedando establecido que todos los agentes participantes serán pertenecientes a esta ciudad. La muestra $n^{\circ} 1$ aborda a ocho Museos de Arte y otras tres instituciones culturales del área, que poseen un desarrollo en sus áreas educativas. La muestra $\mathrm{n}^{\circ} 2$ aborda a cuarenta docentes del subsector de Artes Visuales que realicen o hayan realizado clases en escuelas o liceos de Santiago.

El instrumento utilizado para la recolección de datos es la entrevista semiestructurada, de esta forma permite recabar la mayor información posible sobre las experiencias y percepciones de los actores, profundizando sobre su realidad condescendiendo una flexibilidad al momento de intervenir en miras a obtener una información más completa.

\section{Instituciones Museísticas}

Los museos que se estudian para esta investigación pertenecen a diversas dependencias administrativas y también poseen distintos niveles en torno a la oferta educativa. 
Durante el segundo semestre de 2011 se realizaron las entrevistas a los encargados del área de educación de los museos, encuentros centrados en conocer aspectos ligados a la constitución, funciones y oferta de actividades en el área de educación.

También se incluyen en la investigación tres instituciones que no se enmarcan en la categoría de museos, ya que no poseen colección permanente de obras artísticas, pero que sin embargo realizan una importante labor educativa en el área de las artes visuales realizando oferta educativa de manera constante en sus exposiciones.

Tabla 1 - Museos e Instituciones de la muestra.

\begin{tabular}{|l|l|l|}
\hline Museos / Instituciones & Tipo de Colección & Dependencia \\
\hline Museo de Arte Comtenporaneo MAC & Arte Contemporáneo & Universitario. \\
\hline Museo de Arte Precolombino & Arte Precolombino & Privado \\
\hline Museo Nacional de Bellas Artes & $\begin{array}{l}\text { Arte Chileno y } \\
\text { Extranjero }\end{array}$ & Estatal \\
\hline Museo de Artes Visuales MAVI & $\begin{array}{l}\text { Arte Contemporáneo } \\
\text { Chileno }\end{array}$ & Privado \\
\hline Museo Colonial San Francisco & Arte Sacro & Religioso \\
\hline $\begin{array}{l}\text { Museo de la Solidaridad Salvador } \\
\text { Allende }\end{array}$ & Arte Contemporáneo & Privado \\
\hline Museo de Artes decorativas & Artes Decorativas & Estatal \\
\hline Museo Ralli & Arte Latinoamericano & Privado \\
\hline Museo Artequin & $\begin{array}{l}\text { Reproducciones } \\
\text { Artísticas }\end{array}$ & Privado \\
\hline Centro Cultural Palacio la Moneda & Sin colección propia & Estatal \\
\hline Fundación Telefonica & Sin colección propia & Privado \\
\hline
\end{tabular}

Fuente: Elaboración propia.

La categorización que hemos asignado a través del análisis de las entrevistas, nos ha permitido clasificar en tres categorías, en donde el principal indicador es el desarrollo que poseen en cuanto a la labor educativa. Estas corresponden a los siguientes niveles: Avanzado, Intermedio, y Nulo, los cuales han sido establecidos tomando como base parámetros que están asociados al desarrollo del área educativa de cada uno de ellos.

En torno a la clasificación debemos hacer la distinción de que esta corresponde a una realidad nacional-local basada en el contexto y el desarrollo actual de los museos chilenos.

Instrumento de medición para analizar Museos e Instituciones (parámetros): 
Tabla 2 - Instrumento de Medición para analizar Museos e Instituciones.

\begin{tabular}{|c|c|c|}
\hline Avanzado & Intermedio & Nulo \\
\hline $\begin{array}{l}\text { Posee área educativa } \\
\text { establecida. } \\
\text { Construye } \\
\text { didáctico } \\
\text { Sala didáctica/ Taller } \\
\text { estudiantes } \\
\text { Equipo de guías o } \\
\text { mediadores de audiencia } \\
\text { estables } \\
\text { Realiza Itinerancias. } \\
\text { Capacitación } \\
\text { docentes } \\
\text { Bases de datos, web, } \\
\text { diarios } \\
\text { Genera } \\
\text { metodologías de trabajo en } \\
\text { torno a la educación }\end{array}$ & $\begin{array}{l}\text { Posee área educativa } \\
\text { establecida } \\
\text { Construye Material didáctico } \\
\text { ocasionalmente. } \\
\text { Posee espacio con función } \\
\text { didáctica. } \\
\text { Realiza talleres a estudiantes. } \\
\text { Posee equipo de guías } \\
\text { estables. } \\
\text { Capacitaciones docentes de } \\
\text { manera incipiente. }\end{array}$ & $\begin{array}{l}\text { No posee área educativa } \\
\text { No realiza material } \\
\text { didáctico. } \\
\text { No realiza talleres ni } \\
\text { cursos para docentes. } \\
\text { No posee guías. }\end{array}$ \\
\hline
\end{tabular}

Fuente: Elaboración propia.

\section{Docentes de educación artística}

Paralelamente se entrevistó a cuarenta profesores de arte con el fin de de reconocer e identificar de qué forma los docentes de artes visuales utilizan a los museos de arte. Se intentó realizar una muestra heterogénea a cuanto a género, edad, formación y experiencia docente de los profesores, con el fin de que esta tuviera la amplitud necesaria para estudiar el fenómeno que nos ocupa.

\section{Gráficos - Algunos aspectos relevantes de la muestra.}

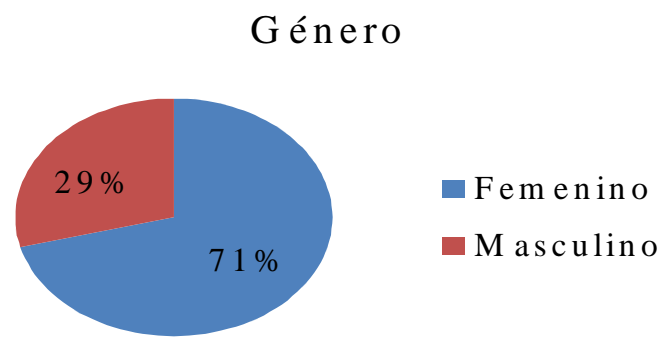




\section{Formación Profesional}

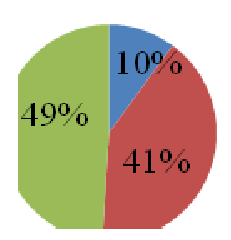

- Licenciado

Licenciado y

Pedagogía

- Pedagogía

Rango Etario

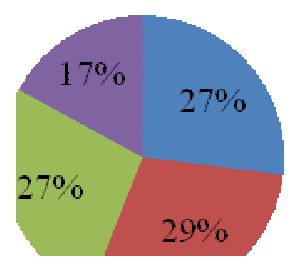

$$
\begin{aligned}
& \square 0 \text { a } 29 \text { aíos } \\
& \square 0 \text { a } 39 \text { alíos } \\
& 40 \text { a } 49 \text { años } \\
& \square 50 \text { o mas arios }
\end{aligned}
$$

Años de Docencia

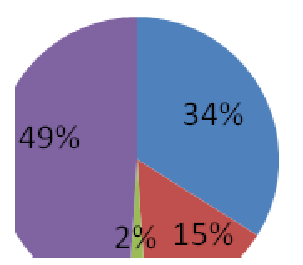

$$
\because 0 \text { a } 3
$$$$
4 \text { a } 7
$$$$
\text { - } 8 \text { a } 10
$$

"11 o más

Tipo de Colegio

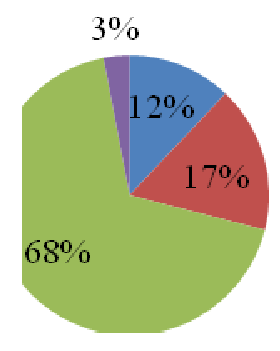

Particular

Municipal

Particular

Subvencionado

a.Jubilado

Fuente: Elaboración propia.

Dentro de los aspectos que se consultaron en la entrevista semi-estructurada podemos destacar los siguientes tópicos:

-Asistencia a los museos de arte con los alumnos (cantidad de veces al año);

-Canales de información utilizados para conocer las opciones educativas que ofrecen los museos; 
-Pertinencia de la visita a un museo y beneficios que considera esta otorga a los estudiantes;

-Canales de comunicación entre profesor de arte y educador de museo en miras a orientar la visita a determinados aprendizajes;

-Asistencia a capacitaciones para profesores ofertadas por los museos.

\section{Resultados obtenidos}

En el área del desarrollo de la función educativa se pudo categorizar a los museos estudiados en función a los parámetros establecidos en el instrumento de medición para analizar museos e instituciones.

Esta categorización otorga resultados optimistas en torno al creciente desarrollo de la función educativa de los Museos de Arte de Santiago de Chile, la cual se ha incrementado de forma sustantiva durante los últimos años logrando revertir el estancamiento se mantuvo durante décadas en esta área.

Este impulso se relaciona directamente con el retorno a la democracia en el año 90, la inyección de recursos por parte del estado y la creación de $\operatorname{CECA}^{3}$ Chile.

Tabla 3 - Clasificación de Museos

\begin{tabular}{|c|c|c|c|}
\hline Categorías & Avanzado & Intermedio & Nulo \\
\hline $\begin{array}{l}\text { Museos / } \\
\text { instituciones }\end{array}$ & $\begin{array}{l}\text { Museo Nacional de Bellas Artes } \\
\text { Museo de Artes Visuales (MAVI) } \\
\text { Museo de Artes Decorativas } \\
\text { Museo de Arte Contemporáneo } \\
\text { (MAC) } \\
\text { Artequín } \\
\text { Fundación Telefónica } \\
\text { Centro Cultural Palacio la Moneda }\end{array}$ & $\begin{array}{l}\text { Museo Colonial San } \\
\text { Francisco } \\
\text { Museo de Arte Precolombino } \\
\text { Museo de la Solidaridad } \\
\text { Salvador Allende }\end{array}$ & Ralli \\
\hline
\end{tabular}

Fuente: Elaboración propia.

\section{Docentes}

${ }^{3}$ CECA-Chile es el Comité de Educación y Acción Cultural, es uno de los comités más relevantes establecidos por ICOM y en Chile se constituye en 2005, convirtiéndose en una entidad muy importante en el impuso de la función educativa de los museos chilenos. Dentro de sus actividades más relevantes se encuentra la organización del congreso de Educación Museos y Patrimonio realizado en Santiago de Chile de forma bianual desde 2005 . 
Algunos de los datos relevantes que hemos obtenido a partir del análisis de las entrevistas es la notoria diferencia entre los docentes que utilizan los museos y los que no. Los de menor edad utilizan mayoritariamente los museos y lo hacen con el fin de actualizar conocimientos.

Respecto a la frecuencia hemos podido establecer que fluctúa entre cero y tres visitas al año, existiendo un panorama bastante heterogéneo en torno a la planificación y calidad de estas visitas. Existen colegios que organizan visitas a instituciones sin consultar la opinión del profesor, programándolas sin considerar los aspectos académicos y la pertinencia de la visita. Esto genera que la salida al museo esté desvinculada al área convirtiéndose en un "paseo", más que visita con un fin pedagógico.

Esto último se relaciona con un punto fundamental en torno a la pedagogía museística, la actual oferta de actividades específicas para los visitantes escolares se realiza con el objetivo de generar una conexión con los establecimientos educacionales y docentes, alianza que no sólo significa apertura y facilidades para asistir sino la intención de conectar de forma significativa ambas esferas educativas, esto implicaría comunicación previa entre docente y museo y planificación conjunta que permita optimizar la visita, aspecto que en nuestra investigación hemos podido establecer que no siempre se da en una forma efectiva.

Respecto al acceso de información a las actividades de los museos podemos notar que esta también se relaciona con la edad del profesor, siendo los más jóvenes quienes se informan a través de correo electrónico, web y redes sociales y los docentes de mayor edad a través de la difusión mediante afiches, correo postal, prensa y finalmente traspaso de información entre colegas y por último el contacto telefónico directo con los museos.

Esto le permite a los museos comprender que aún es necesario continuar la difusión por los medios tradicionales si es que se desea llegar a un espectro mayor de docentes, debido a que no todos utilizan de forma activa los medios digitales.

Respecto al momento en que los docentes consideran apropiado visitar el museo, los profesores consideran que es puede ser pertinente asistir a un museo en las tres instancias del proceso educativo, señalando el inicio o motivación, desarrollo y finalmente cierre de las actividades como instancias claves en donde una visita puede ser beneficiosa para los estudiantes y su aprendizaje, denotando que no hay claridad en torno a la programación de la visita dentro de la unidad didáctica.

Finalmente a través del análisis de las entrevistas podemos señalar que existen diversos factores que influyen para que los docentes no asistan a los museos y se dificulte 
la conexión entre Museo y Escuela. Generalmente dentro de un mismo escenario es más de un factor el que dificulta la formación de dicha alianza, de nuestra muestra se pueden extraer los siguientes como factores principales: Motivación docente y desconocimiento de oferta educativa de los museos, poco interés de establecimiento hacia la educación no formal presentando dificultades para realizar este tipo desalidas, mal comportamiento de estudiantes, largas distancias para trasladarse a los museos, falta de recursos económicos para realizar dicho viaje, desvinculación con los contenidos vistos dentro de la clase, desinterés entre los mismos estudiantes, entre otros factores.

\section{Consideraciones y conclusiones}

Entre las conclusiones más significativas de nuestra investigación, cabe destacar que el nexo que realmente se genera entre museos, instituciones culturales y establecimientos educacionales, se encuentra en una etapa de desarrollo, pues ambas esferas educativas, tanto la de los museos e instituciones culturales, como la de los docentes, han desarrollado estrategias y metodologías para fortalecer este nexo creando alianzas desde el punto de vista educativo.

Hoy en día no se concibe que un museo o centro cultural no posea un área o departamento relacionado con educación, lo cual pone de manifiesto la importancia que esta función tiene en el desarrollo de los museos en la actualidad. Esto ha tenido un impacto también en las escuelas donde se comienza a abrir a la opción de que se pueden integrar estas instancias de educación no formal con el fin de enriquecer el proceso de enseñanza-aprendizaje.

Por lo mismo es importante continuar en esta senda y comprender que esta visita al museo, que en muchos casos es la primera para los estudiantes, puede contribuir a la apertura de la experiencia de ver y sentir el arte y como una opción real de romper el círculo y el pensamiento tradicional del museo, como un espacio de elite cultural, generando una nueva conciencia y propiciando un acercamiento más directo y constante con estos espacios, entendiéndolos como verdaderos lugares de aprendizaje directo del arte. 
ABSTRACT: The present work focuses on the teaching of visual arts in Santiago, Chile, and the way in which teachers relate with the educative function of Art Museums. Nowadays, the school has lost its key role in teaching since it is no longer thought of as the central nucleus of knowledge due to massive Internet access, mass media and formal and informal initiatives which are crucial in the teaching-learning process. One of these institutions is the art museum, which has developed a powerful teaching mission in the last decades. This work is the result of the research "Art museums and teaching, alliances among museums and teachers of Arts" and centres on studying the relationship between the museum of art and artistic education at schools. In order to establish those relations, a qualitative methodology was used through semi-structured interviews to forty teachers, eight museums and three institutions that have given a great contribution to non-formal art teaching. The results were analysed in an interpretative-descriptive way so as to have meaningful conclusions about the real nexus among art teachers, museums and cultural institutions.

KEYWORDS: Art teaching. Non-formal education. Art. Museums.

\section{REFERÊNCIAS}

BOURDIEU, P. Capital Cultural, escuela y espacio social. Buenos Aires: SiglO Veintiuno, 2002.

ICOM. International Council of Museums. Definición del Museo. Disponible en: <http://icom.museum/la-vision/definicion-del-museo/L/1/>. Acceso en: 30 jul. 2012. 